\title{
Virtual Team Governance: Addressing the Governance Mechanisms and Virtual Team Performance
}

\author{
Yihong Zhan ${ }^{1}, Y_{u} \mathrm{Bai}^{2}$,Ziheng Liu ${ }^{3}$ \\ 1 Graduate Department, Central China Normal University, \\ zyh@mail.ccnu.edu.cn \\ 2 Information Management Department, Central China Normal \\ University, Baiyu926@sohu.com \\ 3 The Department of Information Technology, Central China \\ Normal University,Lzh20201@yahoo.com.cn
}

\begin{abstract}
As technology has improved and collaborative software has been developed, virtual teams with geographically dispersed members spread across diverse physical locations have become increasingly prominent. Virtual team is supported by advancing communication technologies, which makes virtual teams able to largely transcend time and space. Virtual teams have changed the corporate landscape, which are more complex and dynamic than traditional teams since the members of virtual teams are spread on diverse geographical locations and their roles in the virtual team are different. Therefore, how to realize good governance of virtual team and arrive at good virtual team performance is becoming critical and challenging. Good virtual team governance is essential for a high-performance virtual team. This paper explores the performance and the governance mechanism of virtual team. It establishes a model to explain the relationship between the performance and the governance mechanisms in virtual teams. This paper is focusing on managing virtual teams. It aims to find the strategies to help business organizations to improve the performance of their virtual teams and arrive at the objectives of good virtual team management.
\end{abstract}

\section{Introduction}

The virtual environment and various communication technologies have created a new context for leadership and teamwork. As technology has improved and collaborative software has been developed, virtual teams are becoming a more common type of work unit and play an increasingly role in organizations [1]. Virtual

Please use the following format when citing this chapter:

7han, Y., Bai, Y., Liul, 7., 2007, in IFIP Intenational Federation for Information Processing, Volume 252, Integration and Innovation Orient to L-Society Volume 2, eds. Wang, W., (Boston: Springer), pp. 282-288. 
teams with geographically dispersed members spread across diverse physical locations have become increasingly prominent. Virtual team is supported by advancing communication technologies, which make virtual team able to largely transcend time and space and to work together and accomplish projects or goals with the combining of the skills of different members in different locations and organizations with different functions [2].

Virtual teams have changed the corporate landscape, which are more complex and dynamic than traditional teams since the members of virtual teams are spread on diverse geographical locations and their roles in the virtual team are different. Therefore, how to realize good governance of virtual team and arrive at good virtual team performance is becoming critical and challenging. Good virtual team governance is essential for a high-performance virtual team. This paper explores the performance and the governance mechanism of virtual team. It establishes a model to explain the relationship between the performance and the governance mechanisms in virtual teams. This paper is focusing on managing virtual teams. It aims to find the strategies to help business organizations to improve the performance of their virtual teams and arrive at the objectives of good virtual team management.

\section{Virtual Team Governance Mechanisms}

Virtual teams are heavily dependent on the advanced information and communication technology. The most distinctive features of virtual teams are that they cross boundaries of space and that teams interaction is mediated through communication technology, for example e-mails, videoconferences and so on. The formation of virtual team facilities companies of various kinds of knowledge and expertise collected in the group working process [3]. Virtual team is becoming common in companies. Thus how to realize good virtual team governance and arrive at good virtual team performance have become critical in organizations. Virtual teams can help organizations to harness, integrate and apply knowledge and expertise that are distributed across organizations and in pockets of collaborative networks [4]. Good management of virtual team is an important orientation of business organizations. In virtual team governance the following mechanisms are of importance in improving the performance of virtual teams: control, trust and cooperation.

\subsection{Control Mechanism}

Control is a helpful mechanism to realize efficient performance of routine partitioned tasks in virtual team work. In virtual team work, there are various kinds of abovementioned routine tasks, for example, regular videoconference, and regular online report and so on. Control is an important mechanism that may help to ensure confidence in cooperative behavior in virtual team. Control mechanism is generic policies or activities that may generate different levels of control. The level of control has a direct influence on the level of trust within a virtual team [5]. Ensuring 
control is quite difficulty, since both high-level and low-level control can result in the dysfunction of virtual team and loss of the advantages of virtual team. Control mechanism can limit the performance of innovation capability in virtual teams as well. It should be applied with some other mechanisms in virtual team management.

\subsection{Trust Mechanism}

Trust is a complex, multifaceted phenomenon, which can function as a highly effective coordinating mechanism in virtual team management. Trust is selected as an important affect-based focus in virtual team management because of its importance to team member perceptions and behaviors towards their team and its importance to individuals working as part of a virtual team. Virtual team is of high dynamics and flexibility, which requires high levels of mutual trust and cooperation in virtual team work and management. It is impossible to monitor and control geographically distributed employees in the virtual team [5]. In virtual team case, trust is a good mechanism to manage people who work in different locations with the help of modern communication technology if companies want to enjoy the benefits of virtual teams. The feature of being virtual requires trust mechanism to make virtual team work. Trust can also help to improve relationships, mutual understanding and knowledge sharing in the virtual team. But ensuring trust is difficult. In virtual team governance, having too much trust can be dysfunctional. In virtual team governance, trust should be combined with control, which can mitigate the weakness of low-trust in control mechanism.

\subsection{Cooperation Mechanism}

In virtual team all the members are working based on the cooperation with different partners in different locations. The members of virtual team are not only dependent on each other, but also responsible for each other. The members of virtual team are supporting each other and helping each other in their work in the virtual environment. Cooperation mechanism helps virtual team in organizations to enhance the competitive strategies of their businesses by cooperation, which can increase the flexibility, responsiveness and cost efficiency as well as the reduction of operational and transactional costs in working process in organizations. Cooperation is perceived as a strategic drive aimed at improving the internal and external resource utilization and the overall profitability of organizations as well [6].

Cooperation mechanism is always used with the trust mechanism in virtual team management. With the advent of cooperation in virtual teams, direct control is no longer so important. Instead trust serves as an important aligning mechanism for geographically dispersed workers. Without trust, virtual teams could not be effective. Individual members in virtual team can cooperate together only if mutual confidence or trust is present among the members in the virtual team [7]. Just as discussed above, the three different governance mechanisms - control, trust and cooperation mechanisms, should be applied in virtual team management together, not only one mechanism, which can help organizations to take advantages of the three different 
governance mechanisms and minimize the disadvantages of them, meanwhile to realize the good performance of virtual team.

\section{Performance of Virtual Team}

There are many potentially important factors that could impact the performance of virtual teamwork in various ways. In this paper we address four aspects of the performance of virtual team. We examine the knowledge management capability, communication capability, shared understanding capability and innovation capability of virtual team to explore the performance of virtual team. Good performance and capability of virtual team is the goal of organizations. Different working capabilities of virtual teams play important roles in the strategic positioning of organizations' performance and the efficiency of virtual team.

\subsection{Knowledge Management}

Knowledge management is an important aspect to evaluate the performance of virtual team. In virtual team knowledge management consists of knowledge learning and knowledge sharing. As above-mentioned, organizations aim at harnessing, integrating and applying knowledge and expertise that are distributed across organizations through virtual team. In virtual team working, knowledge sharing and knowledge learning is the basic requirement for all members in virtual team, which is important for cooperation in virtual team and for the successful utilization of new knowledge produced in the virtual working process [4]. Knowledge management is the prerequisite to transform the knowledge collected and created in virtual team work into the asset of organization, which can influence the efficiency and performance of virtual team.

\subsection{Communication}

In virtual team, all the members, who are working together based on modern information technology, are located in different locations. The members never or rarely meet face-to-face in their working process. Thus communication based on modern technologies becomes quite vital for the collaboration in virtual team. The communication methods in virtual team include videoconference, text-based chat and sharing, and face-to-face meeting, though face-to-face meeting rarely happen in virtual team [8]. Communication is the basic working tool in virtual team. Good communication capability can help virtual team to improve their efficiency on ideas, knowledge and skills exchange, improving understanding among members, producing more new knowledge, and making innovation. In a word, communication based on modern information technology is the basic thing what the members in virtual team should do. 


\subsection{Shared Understanding}

Shared understanding in virtual team is more than a common goal that all the members in the virtual team recognize. Shared understanding involves embracing the strategic direction of a team, including an understanding of the knowledge and expertise each member possesses the role of different members in the team, and how they should interact with each other in order to realize the overall goal of the team. Shared understanding in virtual team can help to establish the cooperation relationship in the entire working process and make the virtual team relationship work very well [2]. It can also encourage the members in virtual team to care more about the whole team work, but not their specific contribution. Shared understanding can help virtual team to cooperate very well, which is the prerequisite for good performance of virtual team.

\subsection{Innovation}

Innovation capability is quite vital for organizations to achieve competitive advantages in the competition with other organizations. Organizations are aiming at make innovation and achieve superiority on technology, product and market through virtual team [6]. Making innovation is the final goal of virtual team. Good innovation capability means good performance of virtual team.

\section{The Governance Model of Virtual Team}

Based on the above discussion on governance mechanism and performance of virtual team, we establish a governance model of virtual team (See Figure 1). This model illustrated that in virtual team management, the three mechanisms, including trust, cooperation and control mechanism, should be applied together, but their importance in management is different (See Figure 1). Trust is of the highest importance and control is of the less.

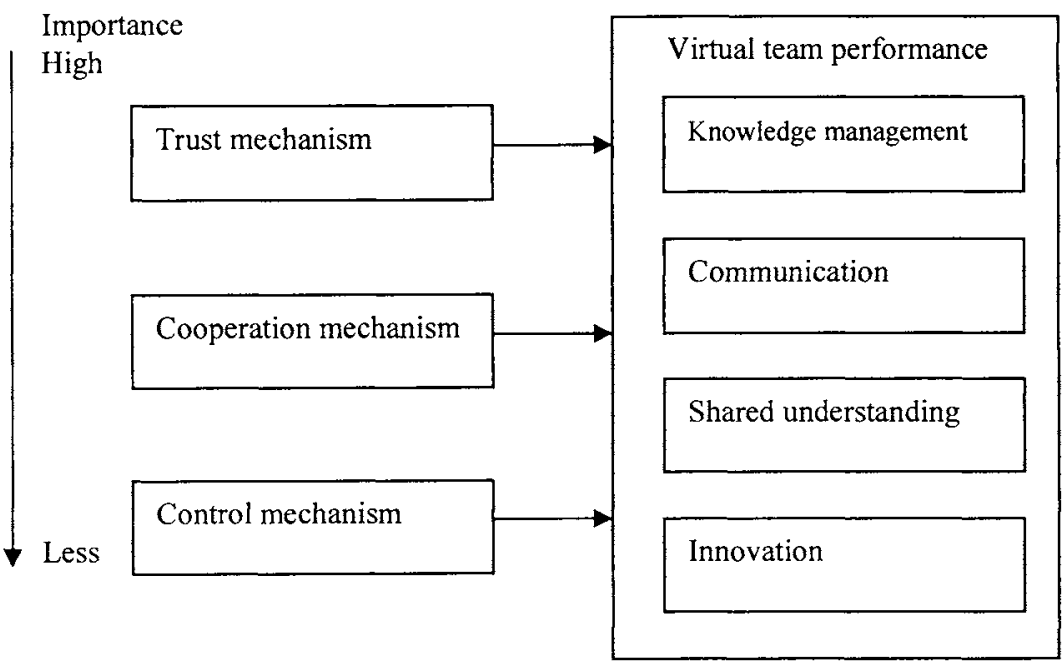


Fig 1. The Governance Model of Virtual Team

Successful application of the three mechanisms in virtual team management can help improve the performance of virtual team, including knowledge management, communication, shared understanding and innovation. For example, trust can encourage the shared understanding among members in virtual team and the knowledge sharing and knowledge learning between each other, which can improve the innovation capability of virtual team. But too much trust might result in business lost as well. In that case control can help to monitor the behavior of members. Over control can limit the innovation capability of virtual team as well. Thus balance the control and trust mechanism is important in virtual team management.

\section{Conclusion}

In this paper some basic concepts and ideas on governance mechanisms and the performance virtual team are discussed. The relationship between the governance mechanism and virtual team performance is explored as well based on a governance model of virtual team. It is concluded that in virtual team management different governance mechanisms should be applied together in order to realize good performance of virtual team, and proper mechanisms applied in virtual team management based on the characteristics of virtual team can improve the innovation capability of virtual team. This paper offers some guide to both practitioners and researchers how to realize good virtual team governance and the prerequisites for virtual team to conduct good performance and reach their business goals.

\section{References}

1. G. Hertel, U. Konradt, and B. Orlikowski," Managing Distance by Interdependence: Goal Setting, Task Interdependence, and Team-based Rewards in Virtual Teams", European Journal of Work and Organizational Psychology 13, 1-28 (2004).

2. L.M. Peters, and C.C. Manz, "Identifying Antecedents of Virtual Team Collaboration", Team Performance Management 13(3/4), 117-129 (2007).

3. H.P. Andres," The Impact of Communication Medium on Virtual Team Group Process", Information Resource Management Journal 19 (2), 1-17 (2006).

4. M. Alavi, and A. Tiwana, "Knowledge Integration in Virtual Teams: the Potential Role of KMS", Journal of the American Society of Information Science and Technology 53(12), 1029-1037 (2002).

5. M.J. Gallivan, "Striking a Balance between Trust and Control in a Virtual Organization: A Content Analysis of Open Source Software Case Studies", Information System Journal 11, 277-304(2001).

6. H. Matlay, and P. Westhead, Innovation and Collaboration in Virtual Teams of Eentrepreneurs: Case Evidence from the European Tourism Industry, Entrepreneurship and Innovation, 8(1), 29-36 (2007). 
7. J.A. Holton, "Building Trust and Collaboration in a Virtual Team", Team Performance Management: An International Journal 7 (3/4), 36-47 (2001).

8. L. A. Hambley, T. A. O'Neill, and T. J.B. Kline, Virtual Team Leadership: The Effects of Leadership Style and Communication Medium on Team Interaction Styles and Outcomes, Organizational Behavior and Human Decision Processes 103, 1-20(2007). 